INPLASY

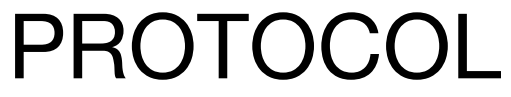

To cite: Silva et al. Decision making in youth team-sports players: A systematic review. Inplasy protocol 202040207. doi:

10.37766/inplasy2020.4.0207

Received: 29 April 2020

Published: 29 April 2020

Corresponding author:

Ana Filipa Silva

anafilsilva@gmail.com

Author Affiliation:

ESDL-IPVC

Support: None.

Review Stage at time of this submission: Data analysis.

Conflicts of interest:

The authors declare no conflict of interest.

\section{Decision making in youth team- sports players: A systematic review}

\author{
Silva, $\mathrm{AF}^{1}$; Conte, $\mathrm{D}^{2}$; Clemente, $\mathrm{FM}^{3}$.
}

Review question / Objective: The aim of this systematic review conducted in the topic of youth team-sports players was three fold: (i) analyze the variations of decision-making processes between young players with different levels of ability; (ii) analyze the variations of decision-making processes between different age-groups; and (iii) analyze the effects of decision-making training based programs on youth players.

Condition being studied: Decision-making in youth team sports players.

Information sources: The search was limited to original articles published online until January 2020. Literature reviews, overviews, conference proceedings, and masters and Ph.D. thesis were excluded.

INPLASY registration number: This protocol was registered with the International Platform of Registered Systematic Review and Meta-Analysis Protocols (INPLASY) on 29 April 2020 and was last updated on 29 April 2020 (registration number INPLASY202040207).

\section{INTRODUCTION}

Review question / Objective: The aim of this systematic review conducted in the topic of youth team-sports players was three fold: (i) analyze the variations of decision-making processes between young players with different levels of ability; (ii) analyze the variations of decision-making processes between different age-groups; and (iii) analyze the effects of decision- making training based programs on youth players.

Condition being studied: Decision-making in youth team sports players.

Rationale: There is an absence of a systematization about the type of decisionmaking studies conducted in youth teamsports players. In team sports, the tactical behavior and the quality of performance is 
closely related with the decisions made by players and for that reason, it is extremely important understand how decisionmaking occurs. Moreover, it is also important to qualify the studies conducted in youth and the main topics and evidence that have.

\section{METHODS}

Search strategy: The search included studies from January 1980 till January 2020 for relevant publications using the following keywords: (youth OR maturation) AND decision-making AND (team sports OR volleyball OR soccer OR futsal OR American football OR football OR basketball OR handball OR rugby OR cricket OR korfball).

Participant or population: Youth team sports players.

Intervention: In training intervention, effects of decision-making based programs were tested in decision-making skills.

Comparator: Age and different levels of ability.

Study designs to be included: Cohort studies or cross-sectional studies.

Eligibility criteria: The inclusion criteria were: (i) decision-making studies in youth players; (ii) decision-making studies in team sports; (iii) only studies comparing different performance levels, players from different age groups and effects of decision-making training programs were included; (iv) only full-articles; (iv) only written in English; (v) article presented an enough information about the sample and experimental approach/procedures (e.g., describe the procedures of data collection, the experimental approach, the instruments and the measures).

Information sources: The search was limited to original articles published online until January 2020. Literature reviews, overviews, conference proceedings, and masters and Ph.D. thesis were excluded.
Main outcome(s): response accuracy; movement accuracy; decision-time; eyesearching strategies.

Quality assessment / Risk of bias analysis: Methodological quality was assessed using the STROBE Statement, which is a 22-item checklist that is considered essential for the accurate reporting of observational studies. This checklist includes a link between the title of the article and its abstract (item 1), introduction (items 2 and 3), methods (items 4 to 12), results (items 13 to 17), and discussion (items 18 to 21) sections, as well as any other information (item 22). Of those, 18 items are common to all three designs, while four of them (items $6,12,14$, and 15) are design-specific, with different versions for all or part of the item. For some items (indicated by asterisks), the information should be given separately for cases and controls in case-control studies or for exposed and unexposed groups in cohort and cross-sectional studies.

Strategy of data synthesis: For the articles included in this study, all authors discussed how the information should be organized regarding the characteristics of the studies and the results of the assessed measurement properties. Afterward, two of the authors extracted data regarding the participants' characteristics (i.e., number, age, and skill level); the study's objective; its design (i.e., its structure); the measures assessed; and the main results.

\section{Subgroup analysis: None.}

Sensibility analysis: None.

Language: English.

Countries involved: Portugal and Lithuania.

Keywords: decision making; youth; sports; team sports; performance.

Contributions of each author:

Author 1 - Ana Filipa Silva.

Author 2 - Daniele Conte.

Author 3 - Filipe Manuel Clemente. 